\title{
Stabilized operation of the Spallation Neutron Source radio-frequency quadrupole
}

\author{
Sang-ho Kim, Alexander Aleksandrov, Mark Crofford, John Galambos, Paul Gibson, Tom Hardek, Stuart Henderson, \\ Yoon Kang, Kay Kasemir, Charles Peters, David Thompson, Martin Stockli, and Derrick Williams \\ Oak Ridge National Laboratory, Oak Ridge, Tennessee 37831, USA
}

(Received 22 March 2010; published 2 July 2010)

\begin{abstract}
The Spallation Neutron Source (SNS) radio-frequency quadrupole (RFQ) had resonance control instabilities at duty factors higher than approximately $4 \%$. Systematic investigations have been carried out to understand the cause of the instability and to ensure the operational stability of the RFQ. The most critical source of the instability is revealed to be an interaction between hydrogen released by beam bombardments and the RFQ rf field resulting in a discharge, which consumes additional rf power and could cause the RFQ to operate in an unstable region. This paper reports improvement of the SNS RFQ operational stability based on the findings during the SNS operation.
\end{abstract}

DOI: 10.1103/PhysRevSTAB.13.070101

PACS numbers: 29.20.Ej

\section{INTRODUCTION}

The Spallation Neutron Source (SNS) front-end (FE) system is composed of an ion source, low energy beam transport (LEBT) section, radio-frequency quadrupole (RFQ) accelerator and medium energy beam transport (MEBT) section as shown in Fig. 1. The SNS FE system was developed and initially commissioned at LBNL and then recommissioned at the SNS site at the full rf duty factor with a lower beam duty factor [1-3]. The 402.5MHz RFQ bunches and accelerates the $65-\mathrm{keV}$ beam from the ion source to $2.5 \mathrm{MeV}$. The design specifications of the RFQ are summarized in Table I. The 3.72- $\mathrm{m}$ long RFQ is a four-vane structure operating in the $\mathrm{TE}_{120}$ quadrupole mode made of 449 cells. The separation between dipole and quadrupole is obtained by using straight rods in both horizontal and vertical directions, called $\pi$-mode stabilizers. The RFQ has two cooling channels; one for the wall sides and the other for the vanes, as shown in Fig. 2. The sensitivity of the resonance frequency to the vane cooling water temperature is approximately $-33 \mathrm{kHz} /{ }^{\circ} \mathrm{C}$ and to the wall cooling water temperature is approximately $+27 \mathrm{kHz} /{ }^{\circ} \mathrm{C}$. The RFQ has six $1300-1 / \mathrm{s}$ cryopumps to maintain a vacuum level in the low $10^{-7}$-torr range with the operation of the ion source. The sensitivity of the resonance frequency to the vane tip dimension is about $43 \mathrm{MHz} / \mathrm{mm}$, which implies that the mechanical stability of the distance between vane tips is very critical not only during the fabrication but also during operations, because a thermal instability can bring the RFQ beyond the allowable control band.

Since the initial commissioning of the accelerator complex in 2006, the SNS has begun neutron production operation and beam power ramp-up has been in progress toward the design goal of 1.4-MW beam on the target. As the design beam power is almost an order of magnitude higher compared to existing neutron facilities, all subsystems of the SNS were designed and developed for substantial performance improvements compared to existing accelerators with some subsystems being the first of a kind. Many performance and reliability aspects of some systems were unknown and unpredictable; therefore it has taken time to understand the systems as a whole and to determine if additional performance improvements are needed [4-6]. The RFQ showed resonance control instabilities when operating at $60-\mathrm{Hz}$ beam and at a pulse length greater than $700 \mu \mathrm{s}$, which was one of the limiting factors in reaching 1-MW beam power. The machine downtime due to this RFQ instability was longer than $30 \mathrm{~min}$ per day, which affected the overall machine availability. In this paper, findings on the RFQ instability and improvements implemented to avoid it will be discussed. With the issues on the operation of the RFQ being long-term stability, observing long-term parameter correlations has been a

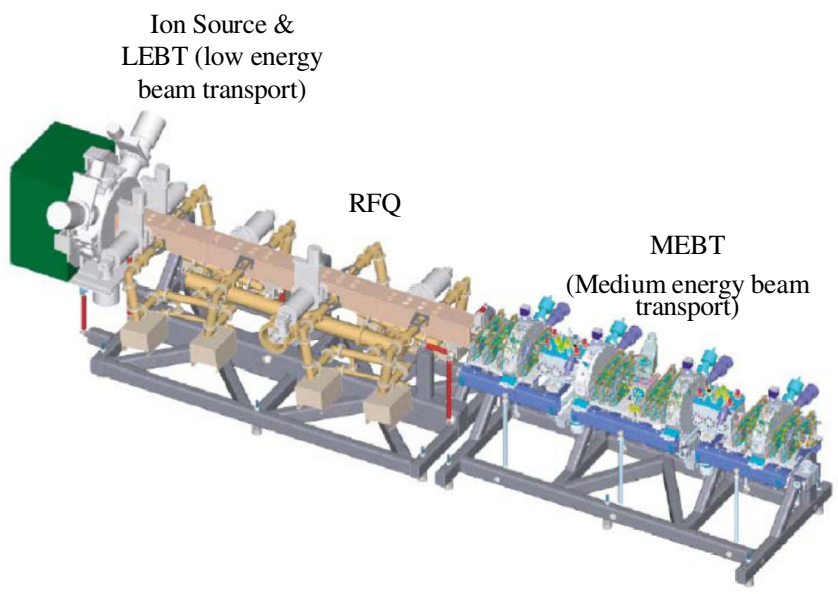

FIG. 1. (Color) The layout of the SNS front-end (FE) system. 
TABLE I. Major parameters of the SNS RFQ.

\begin{tabular}{lc}
\hline \hline Ion species & $\mathrm{H}^{-}$ \\
Input energy & $65 \mathrm{keV}$ \\
Output energy & $2.5 \mathrm{MeV}$ \\
Peak current & $40 \mathrm{~mA}$ \\
Beam pulse length & $1 \mathrm{~ms}$ \\
Repetition rate & $60 \mathrm{~Hz}$ \\
Frequency sensitivity to vane tip displacement & $43 \mathrm{MHz} / \mathrm{mm}$ \\
Frequency sensitivity to vane cooling water temperature & $-33 \mathrm{kHz} /{ }^{\circ} \mathrm{C}$ \\
Frequency sensitivity to wall cooling water temperature & $27 \mathrm{kHz} /{ }^{\circ} \mathrm{C}$ \\
Peak surface electric field & $83 \mathrm{kV}(1.85 \mathrm{Kilpatrick})$ \\
Dipole mode suppression & $\pi$-mode stabilizer \\
\hline \hline
\end{tabular}

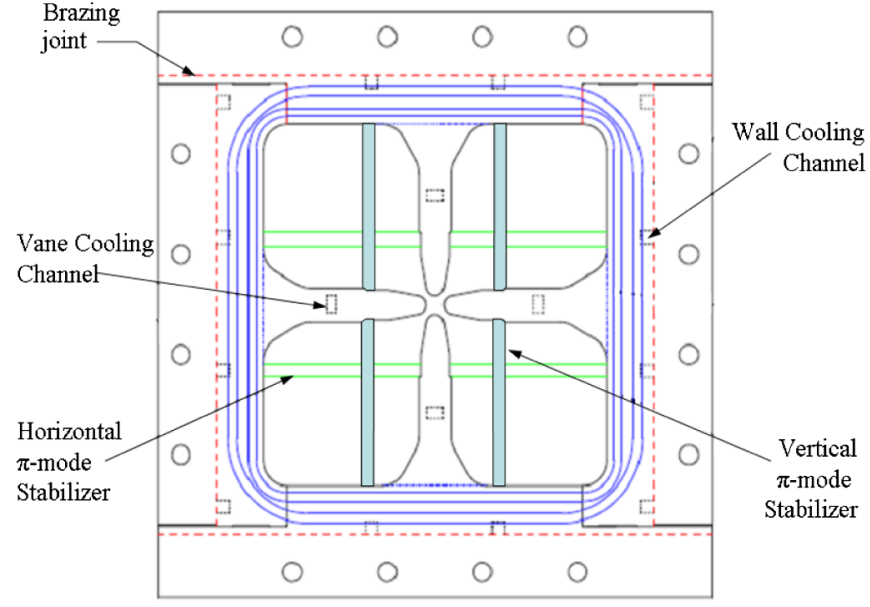

FIG. 2. (Color) Cut view of the SNS RFQ.

valuable tool for understanding the driving mechanisms of the instability.

\section{RFQ INSTABILITY}

When the RFQ enters an unstable region, the typical symptom is that the resonance frequency of the RFQ drops rapidly within a minute by more than $20 \mathrm{kHz}$ and the low level radio-frequency resonance control is lost as shown in Fig. 3, and is sometimes accompanied with structure arcing. We refer to this sudden loss of resonance control as the

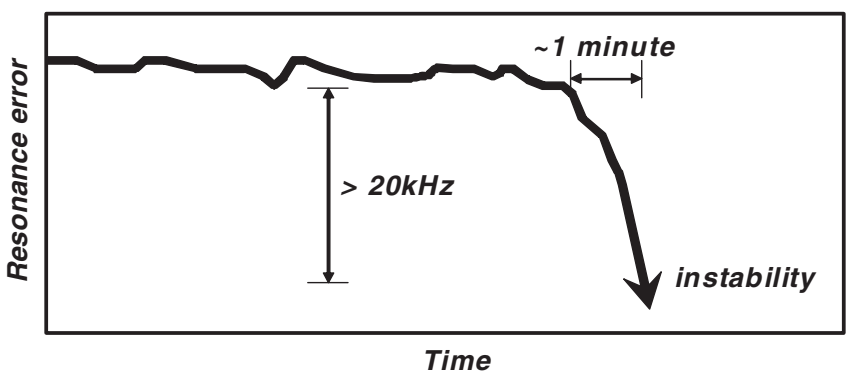

FIG. 3. The typical behavior of the RFQ resonance error when the RFQ shows instability.
"RFQ instability." The resonance error is a purely passive parameter that measures the detuning amount of the RFQ structure primarily determined by thermally induced dimensional changes during operation.

In normal operation when the instability is not present, the rf field regulation and jitter in closed loop operation along with cooling water temperature fluctuations are confirmed to be stable enough for the resonance error fluctuations to be less than $\pm 3 \mathrm{kHz}$. Stability of the water system is better than $\pm 0.03^{\circ} \mathrm{C}$. The long-term rf field stability is better than $0.3 \%$. Both fluctuation levels are too small to explain the large excursions of resonance error and instabilities of the SNS RFQ.

The speed of instability buildup is approximately a minute or less. It is much quicker than the thermal time constant of the RFQ structure, which is about $5 \mathrm{~min}$. After the instability occurred, the outlet temperature of the vane cooling water typically showed a small increment of about $0.2^{\circ} \mathrm{C}$ with a few minutes delay, which is a passive response of water temperature to the instability and indicates an additional thermal dissipation around the vanes. When the vanes get hotter from additional thermal dissipation, the distance between the vanes decreases, which results in increases of capacitance, and the resonance frequency shifts in the negative direction. Since the short-term correlations between related parameters do not clearly indicate causes and consequences, the findings and understandings are summarized through long-term correlations of operational parameters with the instability, as described in the following sections.

\section{FINDINGS}

Since some ion source parameters will be used to explain the RFQ instability, the interfaces and relationships between the ion source and the RFQ beam operation are briefly explained here.

Negative hydrogen ions are generated in a multicusp, 2$\mathrm{MHz}$ rf driven plasma, and are transported to the RFQ entrance through the electrostatic LEBT section. A schematic of the ion source, LEBT and RFQ entrance is shown in Fig. 4. The RFQ vacuum, measured by an ionization 


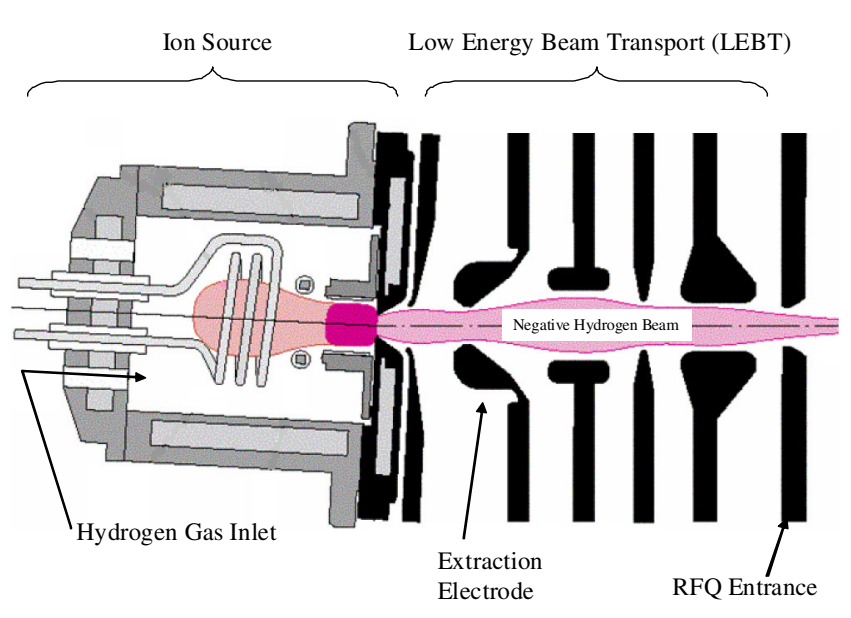

FIG. 4. (Color) Schematics of the SNS ion source, LEBT and RFQ entrance.

gauge located at the wall side of the RFQ, is primarily affected by the hydrogen flow rate in the ion source. After initial RFQ rf conditioning is done, there are no measurable changes from the RFQ vacuum readings with $\mathrm{rf}$ operation. Figure 5 shows the RFQ vacuum readings on the ionization gauge at the upstream end of the RFQ and hydrogen flow rate in the ion source before, during, and after an ion source change. Since changes in the vacuum pressure mostly come from the hydrogen, the actual pressure should be divided by a multiplication factor of the ionization gauge for hydrogen gas which is about 0.56 .

With the ion source energized to $-65 \mathrm{kV}$, negative hydrogen ions $\left(\mathrm{H}^{-}\right)$are accelerated towards the extractor. Two electrostatic lenses refocus the $\mathrm{H}^{-}$beam into the RFQ. Hereafter, $65-\mathrm{kV}$ beam entering into the RFQ is referred to as the "source beam." The beam on/off control

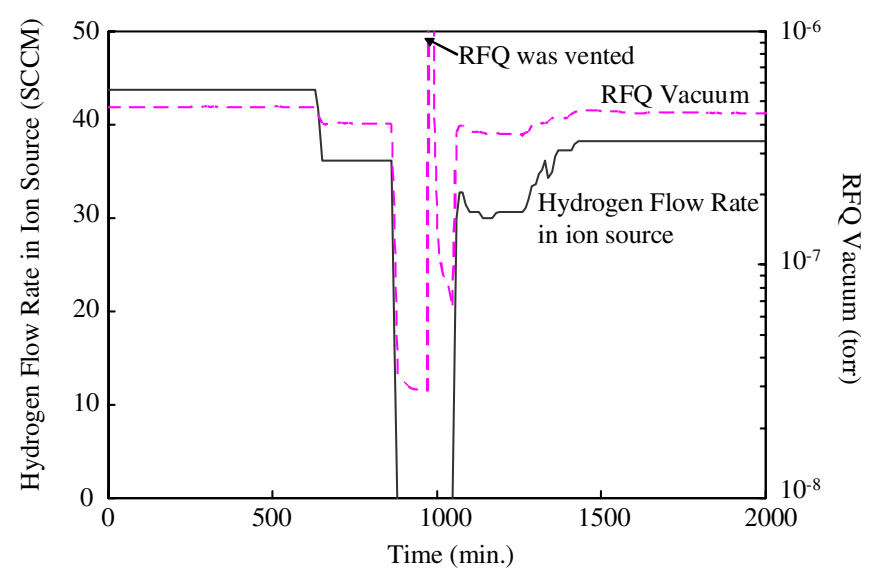

FIG. 5. (Color) The relations between hydrogen flow rate in the ion source and the RFQ vacuum from the ionization gauge at the wall side of the RFQ. The pressure spike in the middle of the plot corresponds to the venting of the RFQ for the ion source maintenance works. The starting time of this plot is 5:30 PM on March 15 in 2009. (a) Beam off

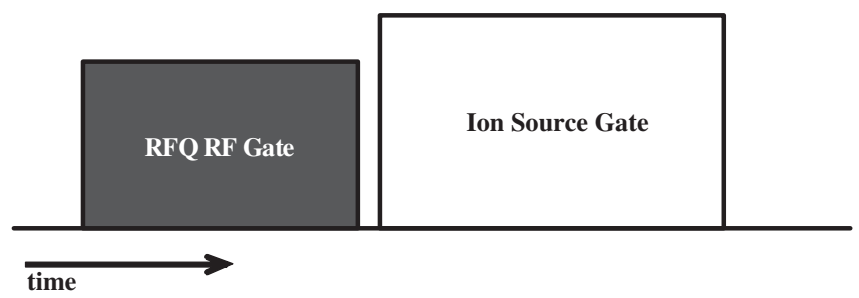

(b) Beam on

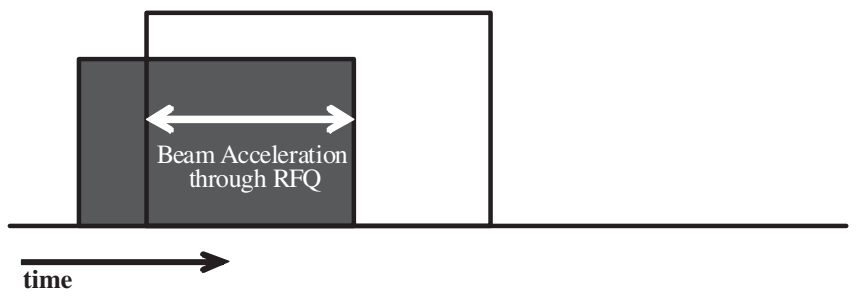

FIG. 6. Timing gate configurations for beam-on and beam-off states after the RFQ.

is achieved by shifting the timing for the RFQ rf gate and the ion source 2-MHz rf gate as indicated in Fig. 6. During the beam-off state as shown in Fig. 6(a), the source beam is dumped into the upstream end of the RFQ after the RFQ rf pulse has occurred. The source beam is normally running at $60 \mathrm{~Hz}$ for neutron production. During beam tuning or beam study we operate $1-\mathrm{Hz}$ beam, in which case 59 pulses out of 60 pulses per second are in the beam-off state. Additionally, some portion $(<10 \%)$ of the beam in the beam-on state will be lost in the RFQ by design.

A few observations will be introduced for the discussions in the next session. The first one is that the net $\mathrm{rf}$ power, which is the difference between forward rf power and reflected $\mathrm{rf}$ power during the $\mathrm{rf}$ pulse, varies at a constant rf field in the closed loop and shows direct correlations with resonance error excursions. The fluctuation of the resonance error is, however, much bigger than the amount that would be expected from fluctuations of operating parameters such as water temperature, rf field, vacuum, source beam condition, etc. Figure 7 is an example that shows the correlation of the net rf power and resonance error during a beam-off state. When net rf power goes up, the resonance frequency moves to a negative direction, which implies that additional $\mathrm{rf}$ power is heating up the vanes. In this example the allowable resonance control band was $\pm 20 \mathrm{kHz}$ and the RFQ did not hit the instability. The amount of the net rf power increment at instability depends on RFQ rf field strength, rf duty factor, and ion source conditions. About $30-\mathrm{kW}$ increments in the net $\mathrm{rf}$ power do not drive the instability in the SNS RFQ. When the resonance error of the structure was more negative it tended to become more unstable, showing sharp dips. Since the speed of the dips is much less than a minute, the water cooling system cannot compensate for this change and can even drive an unstable oscillation due to 


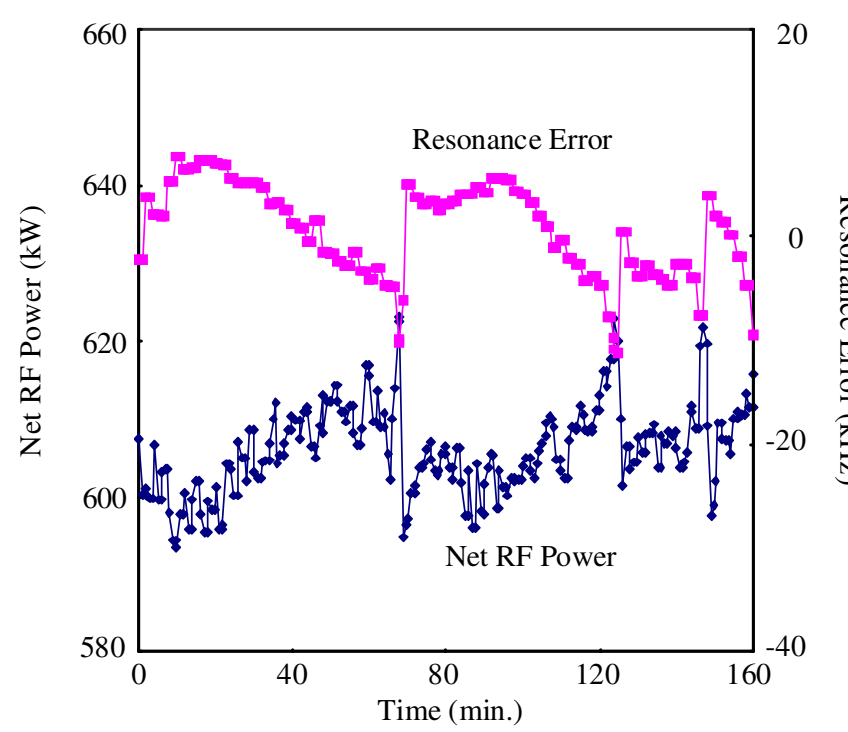

FIG. 7. (Color) Correlations between the resonance error and the net $\mathrm{rf}$ power at the beam-off state and source beam on. The sharp dips in resonance error at around $-10 \mathrm{kHz}$ are precursors of the RFQ instability. All other parameters were stable; Hydrogen flow rate in the ion source is $31.8 \mathrm{sccm}$, RFQ $\mathrm{rf}$ pulse width is $725 \mu \mathrm{s}$, ion source gate width is $836 \mu \mathrm{s}$, RFQ vacuum is $4 \times$ $10^{-7}$ torr, RFQ field strength is 0.351 in arbitrary units, the vane cooling water temperature is $21.2^{\circ} \mathrm{C}$, and the wall cooling water temperature is $21.7^{\circ} \mathrm{C}$. The starting time of this plot is 9:40 AM on April 7 in 2009.

the loop delay of the water system. In order to compensate for these additional $\mathrm{rf}$ load changes and to increase the RFQ operational stability, a slow control loop around the rf pulse width has been implemented. The loop is set to be adjustable over a small range of pulse widths, and additionally the RFQ operation is set to run at the positive resonance error region to help maintain stability. The basic idea of this scheme is to adjust the pulse width, which consequently adjusts the rf load, before the resonance error reaches an unstable region. But the adjustable range of the pulse width is limited by the required beam pulse length and the available high power rf pulse width. This scheme has more meaning as a fine frequency tuning than instability suppression.

The next observation concerns the contribution of the source beam to the RFQ stability either in the beam-on or the beam-off states. At $t=A$ in Fig. 8, the plasma in the ion source extinguished and gas puffing was attempted a few times which corresponds to the small spikes in both hydrogen flow in the ion source and the RFQ vacuum readings. As soon as the source beam was off due to the extinction of plasma, the resonance error jumped up to a more positive value and the net rf power decreased by approximately $40 \mathrm{~kW}$. Hydrogen flow continued until $t=$ $B$ in Fig. 8. To keep the resonance error within the allowable control band, the rf pulse width was manually increased from 745 by $855 \mu \mathrm{s}$. At $t=B$ in Fig. 8, the

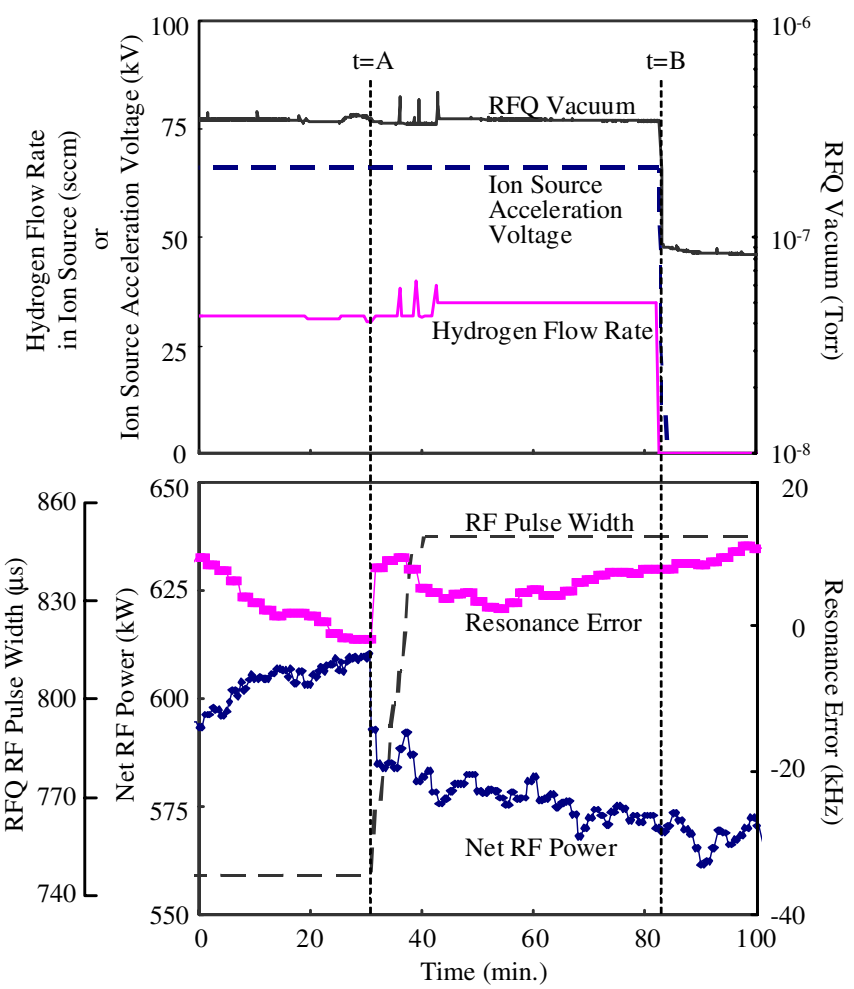

FIG. 8. (Color) The dependencies of the RFQ stability on the source beam at the beam-off state. At $t=A$, ion source plasma was extinguished. At $t=B$, ion source acceleration voltage and hydrogen flow in the ion source were turned off. Ion source gate width is $836 \mu \mathrm{s}$, RFQ field strength is 0.350 in arbitrary units, the vane cooling water temperature is $21.2^{\circ} \mathrm{C}$, and the wall cooling water temperature is $21.7^{\circ} \mathrm{C}$. The starting time of this plot is 3:30 PM on April 7 in 2009.

hydrogen flow in the source turned off, which did not affect the RFQ stability as much as the source beam previously had.

The overall RFQ stability of the beam-on state is about the same as that of the beam-off state or slightly different, depending on the source condition such as alignment of the ion source to the RFQ. Figure 9 is an example of the beamon state, during a period of operation at about $800-\mathrm{kW}$ beam power on the target. In this example the "auto pulsewidth adjustment" was incorporated to compensate for the additional thermal loads. The spike in the resonance error at the very beginning of this plot corresponds to the turn-on of RFQ, which is normal at the turn-on period. The net rf power without beam loading (beam-off state) was $570 \mathrm{~kW}$. With beam-on the net rf power increased to approximately $640 \mathrm{~kW}$. The difference of the net power at the very beginning corresponds to the beam loading of the RFQ. The noisy spikes in the resonance error indicate intermittent beam-off periods whose durations are less than a minute primarily from the machine protection system trips. There were also medium-term beam-off periods ranging from $10 \mathrm{~min}$ to a few hours for various reasons as seen in the beam power plot. The RFQ was stable without any 


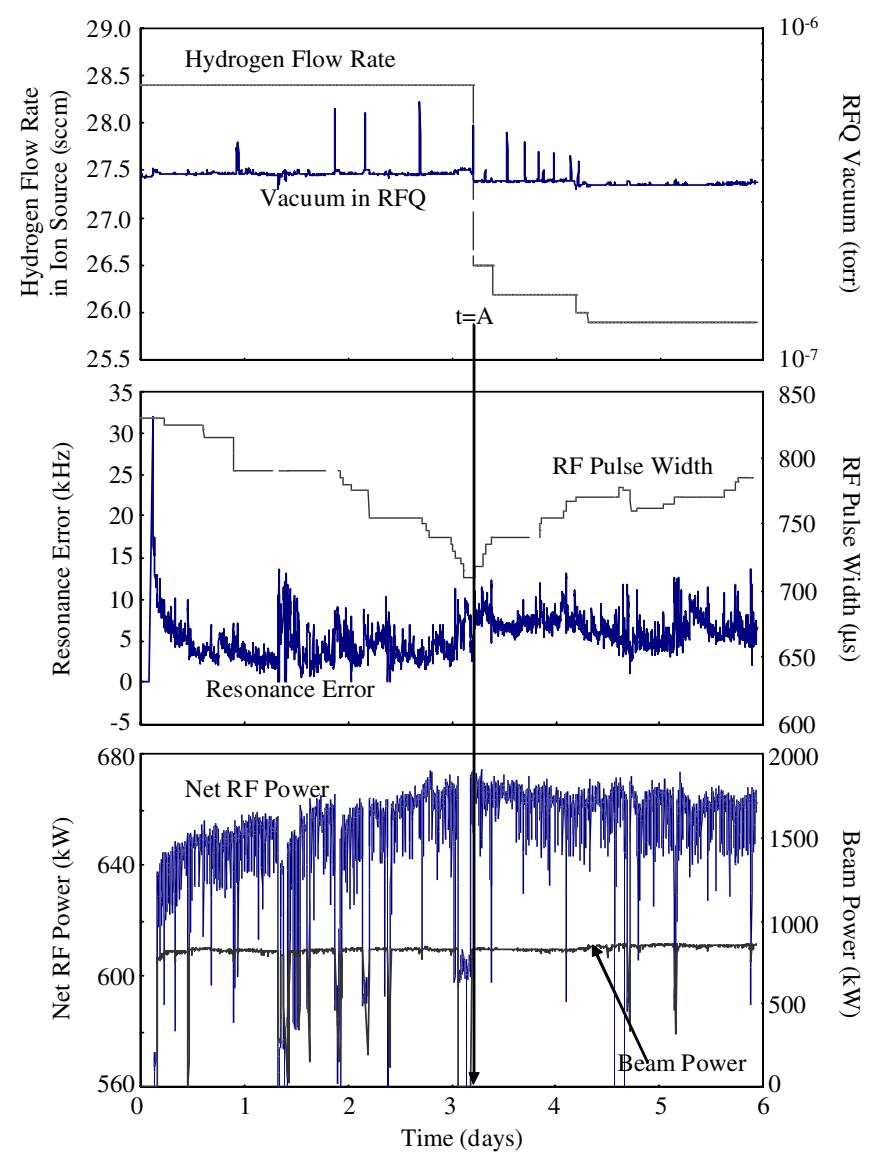

FIG. 9. (Color) The RFQ stability of the beam-on state. The net rf power includes beam loading. Ion source gate width is $836 \mu \mathrm{s}$, average beam current during a macropulse is $24 \mathrm{~mA}$, beam pulse length is $680 \mu \mathrm{s}$, RFQ field strength is 0.350 in arbitrary units, the vane cooling water temperature is $21.2^{\circ} \mathrm{C}$, and the wall cooling water temperature is $21.8^{\circ} \mathrm{C}$. The starting time of this plot is 1:36 AM on April 20 in 2009.

instability-driven downtime during the whole period in this plot which covers six days of operation. The net rf power gradually increased during the first three days until time $t=A$ in the plot. During this time the rf pulse length automatically decreased from 830 to $710 \mu$ s to keep the resonance error in the allowable band where the net $\mathrm{rf}$ power during both the beam-off state and the beam-on state increased from 570 to $600 \mathrm{~kW}$ and from 640 to $670 \mathrm{~kW}$, respectively. The hydrogen flow in the ion source was reduced at $t=A$ in Fig. 9, and this resulted in the net rf power not increasing anymore and also showed a slight decrement while the rf pulse width became wider. The RFQ stability response against the hydrogen flow rate in the ion source is slow; it is, however, clear that the RFQ is more stable at a lower hydrogen flow rate in the long term.

\section{DISCUSSION AND CONCLUSION}

There are a few noticeable observations in the previous sections: (1) direct correlations between variations of the net rf power and the resonance error; (2) immediate responses from the source beam; (3) slow response time but improvements of the long-term stability with lower hydrogen flow rate in the ion source; and (4) more stable operation at positive resonance error.

As shown in the examples in the previous sections, the variation of the net rf power is about $30 \mathrm{~kW}$ during operation at a constant $\mathrm{rf}$ field and constant cooling water temperatures, and goes higher than $40 \mathrm{~kW}$ when the instability occurs. This energy balance cannot be explained either by the direct thermal deposition from the beam or direct interactions between hydrogen gas moving into the RFQ and rf field. The theory for the instability is as follows; hydrogen from the ion source is continuously absorbed on the surface of the RFQ. At the beam-off state $65 \mathrm{kV}$ negative hydrogen beam is dumped around the upstream part of the RFQ, and at the beam-on state some portion $(<10 \%)$ of the negative hydrogen beam will hit the vanes during acceleration by design. Ion beam bombardments are known to be very efficient for gas desorption from metal surfaces [7-9]. The source beam, either at beam-on or beam-off states, enhances hydrogen desorption, which degrades the local vacuum, especially around the vanes even though the vacuum gauge reading at the wall side does not change. Local discharge starts around the vanes where the local vacuum and rf field meet the discharge condition even though it is very mild. This local discharge absorbs the rf power and consequently heats up the vanes, which results in variations of the net rf power at a constant rf field in the closed loop rf control and, consequently, the resonance error changes. Local discharge and vane heating can become even worse if hydrogen desorption increases due to ion beam bombardment and local discharge in the structure. The RFQ can become unstable with a thermal run-away condition causing a sudden decrement of the resonance frequency of the RFQ structure.

To increase the operational stability of the SNS RFQ, the main concern is how to eliminate the discharge condition or at least how to minimize the discharge amplitude. Based on the understandings on the SNS RFQ stability, some operational improvements have been applied.

(1) The hydrogen flow rate in the ion source has been lowered down to the point where stable plasma condition is not affected. This is one of the most important things for the long-term stability since the local vacuum and discharge condition is determined by the amount of hydrogen absorbed on the vane surfaces. If the absorption rate of hydrogen is the same as or smaller than desorption rate by beam bombardments, the net rf power variation will stay constant or decrease. Also a slightly lower rf field is always helpful; however, it should not be lowered to the point where beam quality and beam transmission though the RFQ are affected.

(2) It is difficult to compensate for this kind of fast change in local heating conditions using only adjustments 
of the water temperature. Frequent changes of water temperature to compensate for resonance errors caused by changes of local discharge conditions could give rise to additional feedback instability of the system since the time constant of structure by changing water temperature is quite long. The auto pulse-width adjustment scheme has been added to the low level rf control system to provide fast compensation as required for this fast changing condition, and water temperature is only used for the coarse tuning mechanism.

(3) The allowable range for the resonance error has been shifted to the positive region where the operation is more stable than operation at either zero resonance error or the negative resonance error regions. The SNS RFQ stability at normal operating conditions is primarily determined by the thermal stability of the vanes during the discharge. Operation at a more positive resonance error results in keeping the vane at a colder temperature. The allowable resonance error range is set between -8 to $32 \mathrm{kHz}$, by concerning the operational stability of the RFQ and the allowable rf mismatching.

(4) The stability of the beam-on and beam-off states should be about the same or within a reasonably controllable range. The beam bombardment locations at beam-on and beam-off states are different, which results in different heated regions between the two states. The noisy spikes of resonance errors in Figs. 9 and 10 correspond to the intermittent beam-off state for various reasons during the run such as beam truncation by the machine protection system and other equipment trips. Sometimes alignments between the ion source and the RFQ are not made for the maximum beam transmission to improve the overall beam quality after the RFQ where several more percents of beams are scraped in the RFQ. In these situations the resonance errors drop down by more than $20 \mathrm{kHz}$ at the beam-on state in a few seconds, which implies that more beam bombardments make the stability worse at the beamon state. This observation also supports the theory for the instability. When the alignments are good, the stabilities at both states are well within the controllable range. For a situation where the stability conditions cannot be achievable with this control scheme, a timing system for a different gate width at the beam-off state has been implemented and is ready to use.

Figure 10 shows the operational conditions of the RFQ after the improvements mentioned above were implemented. It shows stable operation for 1-MW beam production on target. The beam pulse length was fixed at $825 \mu \mathrm{s}$ and the RFQ rf pulse width was automatically adjusted between 860 and $920 \mu$ s to maintain stability. In addition, there were a few automatic adjustments of rf pulse width and chiller water temperature during the run period. Since this improvement, the SNS RFQ is running stably without any instability-driven down time. The SNS RFQ has been confirmed to be stable up to $5.5 \%$ rf duty factor with the

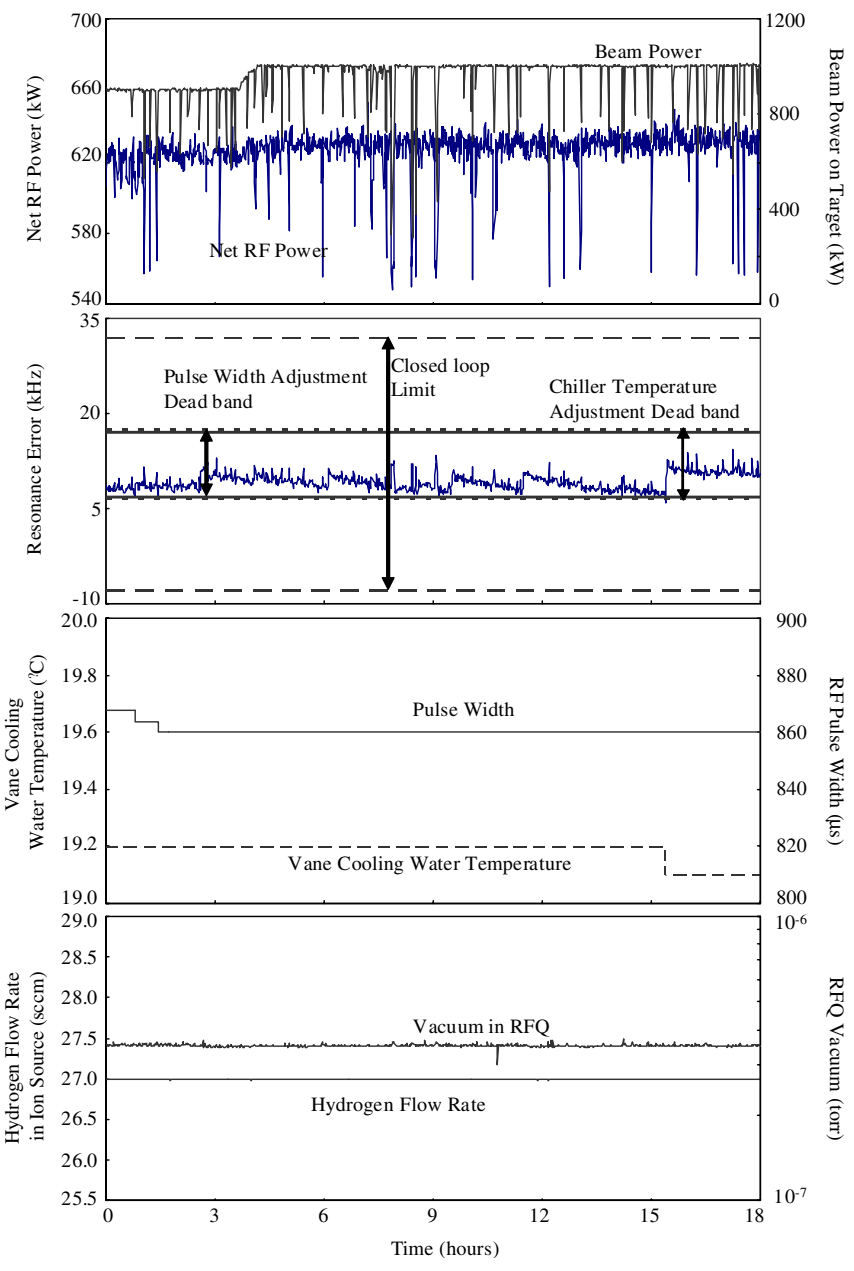

FIG. 10. (Color) RFQ operation for $825 \mu$ s beam after improvements. Ion source gate width is $941 \mu \mathrm{s}$, average beam current during a macropulse is $24 \mathrm{~mA}$ for $900 \mathrm{~kW}$ beam on target and $26.5 \mathrm{~mA}$ for $1000 \mathrm{~kW}$ beam on target, beam pulse length is $825 \mu \mathrm{s}$, RFQ field strength is 0.350 in arbitrary units, and the wall cooling water temperature is $22.3^{\circ} \mathrm{C}$. The starting time of this plot is 12:00 AM on October 23 in 2009.

improvements mentioned above. The operating rf duty factor is currently limited by the high voltage power supply for the RFQ klystron.

\section{ACKNOWLEDGMENTS}

The authors extend our thanks to all our SNS colleagues who contributed to this work, especially to John Mammosser and Mike Plum for the fruitful discussions. SNS is managed by UT-Battelle, LLC, under Contract No. DE-AC05-00OR22725 for the U.S. Department of Energy.

[1] A. Ratti et al., in Proceedings of the Twentieth International Linac Conference, Monterey, CA, 2000 (SLAC, CA, 2000), p. 911. 
[2] R. Keller et al., in Proceedings of the 8th European Particle Accelerator Conference, Paris, 2002 (EPS-IGA and CERN, Geneva, 2002), p. 1025.

[3] A. Aleksandrov, in Proceedings of the 20th Particle Accelerator Conference, Portland, OR, 2003 (IEEE, New York, 2003), p. 65.

[4] S. Henderson et al., in Proceedings of the 10th European Particle Accelerator Conference, Edinburgh, Scotland, 2006 (EPS-AG, Edinburgh, Scotland, 2006), p. 345.

[5] Sang-ho Kim, in Proceedings of the Twenty-fourth International Linac Conference, Victoria, British
Columbia, 2008 (TRIUMP, British Columbia, 2008), p. 11.

[6] M. Plum et al., in Proceedings of the 11th European Particle Accelerator Conference, Genoa, 2008 (EPS-AG, Genoa, Italy, 2008), p. 3560.

[7] I. Kojima et al., Bull. Chem. Soc. Jpn. 53, 2123 (1980).

[8] E. Taglauer and W. Heiland, J. Nucl. Mater. 93\&94, 823 (1980).

[9] E. Taglauer et al., Nucl. Instrum. Methods 168, 571 (1980). 